\title{
The syndemic condition of psychosocial problems related to depression among sexually transmitted infections patients
}

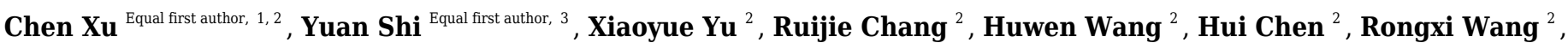 \\ Yujie Liu ${ }^{2}$, Shangbin Liu ${ }^{2}$, Yong Cai ${ }^{2}$, Yang Ni ${ }^{\text {Corresp., }}{ }^{\text {, }}$, Suping Wang ${ }^{\text {Corresp. } 2}$ \\ ${ }^{1}$ Shanghai Skin Disease Hospital, Shanghai, China \\ 2 School of Public Health, Shanghai Jiao Tong University School of Medicine, Shanghai, China \\ 3 Shanghai Jiao Tong University School of Medicine, Shanghai, China \\ Corresponding Authors: Yang Ni, Suping Wang \\ Email address: niyanghj@163.com, wangsuping@shsmu.edu.cn
}

Background. The prevalence of depression in sexually transmitted infections (STIs) patients is much higher than general public. However, studies focusing on comprehensive psychosocial effects on depression among STIs patients are limited. This study aimed to examine associations of multiple psychosocial syndemic conditions with depression among STIs patients in Shanghai, China.

Methods. We conducted a cross-sectional study and recruited 910 STIs patients from Shanghai Skin Disease Hospital. Participants self-reported their demographics and themselves completed the scales of depression, self-esteem, loneliness, social support, entrapment, defeat and interpersonal needs. Logistic regressions were performed to detect the possible contributing psychosocial factors for depression and to verify the syndemic conditions of psychosocial problems.

Results. Of the STIs patient sample, the prevalence of depression was $17.9 \%$. Multivariable analysis showed low-level self-esteem (odds ratio [ORm]: $2.18,95 \% \mathrm{Cl}: 1.19-4.00$ ) and social supports (ORm: 2.18, 95\% Cl: 1.37-3.46), high-level entrapment (ORm: 6.31, 95\% Cl: 3.75-10.62) and defeat (ORm: 2.60, $95 \% \mathrm{Cl}: 1.51-4.48$ ) increased the risk of depression. Psychosocial syndemic conditions magnified effect in fusing depression (adjusted odds ratio [AOR]: 11.94, 95\% Cl: 7.70-18.53). Participants with more than 4 psychosocial problems were about 22 times more likely to have depression (AOR: $22.12,95 \% \mathrm{Cl}$ : 13.19-37.09).

Conclusions. The psychosocial problems syndemic magnifying the risk of depression was confirmed and psychosocial intervention to prevent depression is needed among STIs patients. 
1 The syndemic condition of psychosocial problems related to depression among sexually 2 transmitted infections patients

3

4

Chen $\mathrm{Xu}^{12 \dagger}{ }^{1 \dagger}$, Yuan Shi ${ }^{3 \dagger}$, Xiaoyue $\mathrm{Yu}^{2}$, Ruijie Chang ${ }^{2}$, Huwen Wang ${ }^{2}$, Hui Chen ${ }^{2}$, Rongxi Wang $^{2}$, Yujie Liu ${ }^{2}$, Shangbin Liu ${ }^{2}$, Yong Cai ${ }^{2}$, Yang Ni ${ }^{1 *}$, Suping Wang ${ }^{2 *}$,

${ }^{1}$ Shanghai Skin Disease Hospital, Shanghai 200443, China

${ }^{2}$ School of Public Health, Shanghai Jiao Tong University School of Medicine, Shanghai 200025, China

${ }^{3}$ Shanghai Jiao Tong University School of Medicine, Shanghai 200025, China $\dagger$ Chen Xu and Yuan Shi contributed equally to this work.

Corresponding Author:

Yang $\mathrm{Ni}^{1}$, Suping Wang ${ }^{2}$

Shanghai Skin Disease Hospital, No. 1278, Baode Road, Shanghai 200025, China

Email address: niyanghj@163.com; wangsuping@shsmu.edu.cn

\section{Abstract}

Background. The prevalence of depression in sexually transmitted infections (STIs) patients is much higher than general public. However, studies focusing on comprehensive psychosocial effects on depression among STIs patients are limited. This study aimed to examine associations of multiple psychosocial syndemic conditions with depression among STIs patients in Shanghai, China.

Methods. We conducted a cross-sectional study and recruited 910 STIs patients from Shanghai Skin Disease Hospital. Participants self-reported their demographics and themselves completed the scales of depression, self-esteem, loneliness, social support, entrapment, defeat and interpersonal needs. Logistic regressions were performed to detect the possible contributing psychosocial factors for depression and to verify the syndemic conditions of psychosocial problems.

Results. Of the STIs patient sample, the prevalence of depression was 17.9\%. Multivariable analysis showed low-level self-esteem (odds ratio [ORm]: 2.18, 95\% CI: 1.19-4.00) and social supports (ORm: 2.18, 95\% CI: 1.37-3.46), high-level entrapment (ORm: 6.31, 95\% CI: 3.7510.62) and defeat (ORm: 2.60, 95\% CI: 1.51-4.48) increased the risk of depression. Psychosocial syndemic conditions magnified effect in fusing depression (adjusted odds ratio [AOR]: 11.94, 95\% CI: 7.70-18.53). Participants with more than 4 psychosocial problems were about 22 times more likely to have depression (AOR: 22.12, 95\% CI: 13.19-37.09).

Conclusions. The psychosocial problems syndemic magnifying the risk of depression was confirmed and psychosocial intervention to prevent depression is needed among STIs patients. 
40

41

42

43

44

45

46

47

48

49

50

51

52

53

54

55

56

57

58

59

60

61

62

63

64

65

66

67

68

69

70

71

72

73

74

75

76

77

78

79

80

81

82

Keywords. Sexually transmitted infections, depression, psychosocial problems; syndemic

\section{Introduction}

Sexually transmitted infections (STIs) are spread predominantly by sexual contact and body fluid exchange, among which trichomonas, chlamydia, syphilis, gonorrhea, are most common globally. More than 1 million STIs are acquired every day worldwide(Rowley J June 2019). In China, acquired immunodeficiency syndrome (AIDS), syphilis, gonorrhea are three major types of the greatest prevalence in China. And both AIDS and syphilis showed an upward trend from 2004 to 2016, with an incidence of 3.97/10,000 and 31.97/10,000 in 2016, respectively (Ye et al. 2019). STIs patients are at high risk of HIV infection and transmission due to unprotected sexual encounters. Meanwhile, depressive symptom rates are extremely high among STIs patients. An American study reported $39.2 \%$ of STIs patients in the survey have probable depression. (Erbelding et al. 2001) In 2015, Mo PK et al. reported 43\% probable cases of depression in Chinese female STIs patients in Hongkong (Mo et al. 2015), while the depressed patients account for nearly $4.4 \%$ and $4.2 \%$ of the world and Chinese whole population, respectively (WHO 2017). The issue is increasingly pressing.

For STIs patients, depression and status of infection interact as both cause and effect. Researches have showed that patients with sexually transmitted infections bear heavier psychosocial burden than general population, primarily caused by poor self-image and sexrelated concerns (Qi et al. 2014). Emotional impact is seen most among them. On the other hand, symptoms of depression also concerned with unprotected sexual intercourse, multiple sex partners and giving rise to a potential prevalence of STIs (Hutton et al. 2004; Shrier et al. 2002). Culture difference does not seem to break the correlation. Based on Taiwan National Health Insurance Research Database, Huang et al. conducted a retrospective study in 5,959 depression patients, among which 5.0\% was later diagnosed with STIs, compared to $3.2 \%$ in control group without depression $(\mathrm{P}<0.001)$ (Huang et al. 2018).

Depression is caused by the complex interactions of pathological, social and psychosocial factors (WHO 2020). According to previous studies, people who suffer mental health problems such as low self-esteem, loneliness, unmet social support, entrapment, defeat and dissatisfied interpersonal needs are more vulnerable to depression. Self-esteem has a close relationship with the onset, maintenance, and relapse of depression (Choi et al. 2019; Han \& Kim 2020; Sowislo $\&$ Orth 2013). The relationship between loneliness and depression is a longitudinal effect which is bidirectional but more significant with former as origin (Domènech-Abella et al. 2019). Meanwhile, social support is also significantly correlated with depression and partially mediates loneliness and depression (Liu et al. 2016). The role of defeat and entrapment in depression was first proposed by Gilbert and Allan (1998) and has been confirmed to be promising variables for the study of depression (Carvalho et al. 2013; Gilbert \& Allan 1998; Trachsel et al. 2010). The interpersonal theory of suicide has been applied in prediction of future depression with thwarted belongingness and perceived burdensomeness shown to give contribution to higher interpersonal shame and eventually lead to depression(Carrera \& Wei 2017). The roles of the psychosocial factors above in the development of depression are worth exploring in patients with STIs.

STIs patients may experience multiple mental health problems that may contribute to depression vulnerability in a syndemic way. The concept of syndemic was first proposed by Singer (1996) to describe that several concurring epidemics may synergistically interact to 
83 undermine health in a certain population (Guadamuz et al. 2014; Tsai 2018). Synergistic

84 epidemic or syndemic effect is the aggregation of more than two concurrent or sequential

85 epidemic or disease clusters in a population. The syndemic effect exacerbates the disease burden.

86 The syndemic theory has become increasingly popular for policymaker and program

87 implementers to improve population health, especially in the field of HIV treatment and

88 prevention (Tsai 2018). Previous studies found the combined impacts of psychosocial problems

89 on suicide ideation in HIV+ patients (Wang et al. 2018), men who have sex with men (MSM) (Li

90 et al. 2016) and STIs patients (Wang et al. 2020). Likewise, we hypothesized that there may be

91 magnifying effects of the syndemic of multiple psychosocial factors on depression among STIs

92 patients.

Data reflecting the prevalence of mental health problems, their association with depression and comprehensive effects on depression among STIs patients are limited. The purposes of our study were: 1) to gain in-depth understanding of the STIs patients' mental health status; 2) to construct a comprehensive framework about the combined effect of mental health issues on depression by applying syndemic theory; 3 ) to develop a preliminary psychosocial screening framework to effectively identify the prodrome of depression and to provide theoretical reference for psychosocial intervention strategies among STIs patients.

\section{Materials \& Methods}

102

103

104

105

106

107

108

109

110

111

112

113

114

115

116

117

118

119

120

121

122

123

124

125

126

\section{Participants and Eligibility Criteria}

The cross-sectional study was launched in March, 2018. Patients from two branches (QiuJiang Rd. and BaoDe Rd., Jingan District) of Shanghai Skin Disease Hospital were recruited. Shanghai Skin Disease Hospital is one of the top hospitals specializing in management of STIs, with 136,146 outpatients and 1,845 discharged patients in 2020 ranking first in Shanghai.

Patients either walk-in or hospitalized were invited if they met the following criteria: (1) Aged 18 years or more; (2) clinically diagnosed as all forms of STIs. The STIs were classified into five common diseases (HIV/AIDS, syphilis, gonorrhea, genital warts, genital herpes) and others (Chen 2019); (3) being able to read and sign informed consent and not participating in similar studies in the past six months. The exclusion criterion was participants with mental or cognitive impairment, unconsciousness so that they cannot verbalize their real feelings or fill out questionnaires.

Given that the prevalence of depression among STIs patients is $43 \%$ (Mo et al. 2015), using alpha of 0.05 and a relative error for sampling 0.05 , taking into account a $30 \%$ non-response rate, required sample size was calculated to be 562 . In our study, a total of 910 patients were finally recruited in the study.

\section{Ethics}

The study has been approved by Public Health and Nursing Ethics Committee, Shanghai Jiao Tong University School of Medicine. Background information provided, written informed consent were signed by patients before collection of information. During the process of interview, participants were free to ask any question or to withdraw if they did not want to continue.

\section{Study Procedure}


The cooperation between our study team and Shanghai Skin Disease Hospital was based on a contract signed beforehand with all doctors who worked at STIs department, in-patient and outpatient recruited in the survey. Interviews were carried out by senior medical and graduate students from School of Medicine, Shanghai Jiao Tong University, who had received adequate trainings include in-person review, quality control strategies and privacy protection.

The doctors invited qualified patients to participate in the study. After receiving preliminary approval, the patients were invited to a separate room by the investigators. Our investigators informed participants the study goal, study procedure, potential risks and answered their questions and concerns about the study. Copy of our questionnaire along with the informed consent was provided for each eligible individual. Only after signing the informed consent, can participants complete self-filled questionnaires. A bonus of 80 RMB (US\$12) was given to each person for his/her participation.

\section{Measures \\ Sociodemographic variable}

Sociodemographic variables included outpatient or inpatient, age, gender, education level, marital status, residence status, income, health insurance, sexual orientation, HIV status and type of STIs.

\section{Psychosocial Variables \\ Depression}

The Patient Health Questionnaire-9 (PHQ-9) was adopted as a scale for depression evaluation in STIs patients. This 9-item self-report questionnaire is a widely used screening scale for depression in non-psychiatric settings, such as community, primary care and general hospitals (Spitzer et al. 1999). Severity of each symptom during the previous two weeks is marked from 0 (not at all) to 3 (nearly every day) (Kroenke et al. 2001). A total score (range: $0-27$ ) of $\geq 10$ is the most commonly- recommended cut-point for "clinically significant" symptoms (Kroenke et al. 2010; Manea et al. 2015). Reliability and validity of its Chinese version has been confirmed by prior study(Xing-Chen et al. 2014). The Cronbach's $\alpha$ coefficient of PHQ-9 was 0.91 in this study.

\section{Self-esteem}

Self-esteem was evaluated with Rosenberg Self-esteem Scale (RSES)(Rosenberg 1965), a widely used tool containing 10 items which are scored on 4 points ranging from 0 (strongly disagree) to 3(strongly agree). Higher scores indicated higher levels of global feelings of selfworth (range 0-30) (Sinclair et al. 2010). Any outcome lower than the norm of 15 was regarded as an indication of low self-esteem(Li et al. 2016; Polat et al. 2015). The scale was translated into Chinese by Cheung and Lau(Cheung \& Lau 1985) and was proved to have good reliability and validity(Hu \& Ai 2016). The Cronbach's $\alpha$ coefficient of RSES was 0.84 in this study.

\section{Social support}

Social support from sources of family, friends and significant others was measured by Multidimensional Scale of Perceived Social Support (MSPSS)(Zimet 1988), a 12-item scale. It is scored on a 7-point scale ranging from 1 (very strongly disagree) to 7 (very strongly agree), with a higher total score indicating higher social support (Cronbach's $\alpha=0.95$, range $12-84$ ). The reliability and validity of Chinese version of MSPSS has been verified(Yuan Chen 2018). Any 
173

174

175

176

177

178

179

180

181

182

183

184

185

186

187

188

189

190

191

192

193

194

195

196

197

198

199

200

201

202

203

204

205

206

207

208

209

210

211

212

213

214

215

216

217

218

mark higher than the 25th percentile of 59 would indicate acceptable social support(Li et al. 2016).

\section{Loneliness}

The USL-8 loneliness scale by Hays and DiMatteo was derived from the total 20 original questions at an attempt to shorten survey time and enhance answer quality(Hays \& DiMatteo 1987). Respondents score their level of frequency with 8 statements on a scale ranging from 1 (never) to 4 (always), with higher scores suggesting a higher degree of loneliness. (Cronbach's $\alpha$ $=0.81$, range 8-32). This study uses a Chinese version of the ULS-8 Loneliness Scale. The translated version of the scale was created by Zhou and his colleagues (2012) for their own research on Chinese participants (Zhou et al. 2012). With no exiting recommendation for a cutpoint for USL-8, we defined the 75th percentile of 18 as the cut-point of significant loneliness( $\mathrm{Li}$ et al. 2016).

\section{Entrapment}

Entrapment Scale (ES) with 16 items quantified the escape motivation raised by outside world and internal feelings (Cronbach's $\alpha=0.97$, range 0-64) (Gilbert \& Allan 1998). The response options for each item are "not at all," "a little bit," "moderately," "quite a bit," and "extremely," which correspond to scores of 0-4 (Griffiths et al. 2014). The Chinese version of ES used in this study had good validity and reliability in medical students(Ruijie Gong 2019). Any score higher than 75 th percentile of 20 would be seen as high in entrapment.

\section{Defeat}

The level of defeat was presented by the 16-item Defeat Scale (DS) on ones' thoughts about themselves in the past week (Cronbach's $\alpha=0.92$, range 0-64)(Gilbert \& Allan 1998).

Individuals indicated the extent to their feeling with each of the items on a 5-point rating scale, ranging from 0 (never) to 4 (always)(Griffiths et al. 2014). The reliability and validity of the defeat scale in Chinese had been confirmed in medical students(Hua Tang 2019). Participants who rated a sum higher than 75 th percentile of 23 were classified into high sense of defeat.

\section{Interpersonal Needs}

The 15-item Interpersonal needs questionnaire (INQ-15), designed by professor Van Orden was used to analyze patients' perceived burdensomeness and thwarted belongingness in the past week (Van Orden et al. 2008). Respondents rate how often they feel a certain way ranging from 1 (not at all true for me) to 7 (very true for me). Higher numbers reflect higher levels of perceived burdensomeness and thwarted belongingness (Cronbach's $\alpha=0.85$, range 15-105). The Chinese version of INQ-15 is a valid and reliable measure instrument for assessing interpersonal needs in university students(Xiaomin Li 2015). Likewise, score higher than 75th percentile of 49 was calculated as cut-point to define the dissatisfaction with interpersonal need.

\section{Statistical Analysis}

Statistical analysis was performed via SPSS version 25.0 for Windows. At first, descriptive statistics were adopted to get an overview of sociodemographic characteristic, prevalence of depression, and psychosocial variables. Then, univariate logistic regression was conducted to explore the association between sociodemographic characteristic and depression, between mental health problems and depression. The relationship between all six psychosocial variables and 
219 depression was evaluated though multivariable logistic regression after adjusting for all

220 significant sociodemographic variables. After that, the correlations between psychosocial

221 variables were described by Spearman correlation coefficient. Finally, to examine the

222 psychosocial syndemic effect on depression, different groups were divided based on the number

223 of mental health problems, so that a univariable logistic regression considering all significant

224 sociodemographic variables can be carried out.

\section{Results}

227

228

229

230

231

232

233

234

235

236

237

238

239

240

241

242

243

244

245

246

247

248

249

250

251

252

253

254

255

256

257

258

259

260

261

Sociodemographic characteristic and their associations with depression are presented in Table 1. An overall 910 interviewers completed the survey, the majority (67.5\%) of which were from outpatient clinics. The average age was 38.72 , with a standard deviation of 13.03 . Ratio between male and female was 1:1.08. Of all the participants interviewed, $44.1 \%$ were local and $93.6 \%$ had a health insurance to pay the bill. More than half $(61.8 \%)$ of them were married, followed by $30.4 \%$ unmarried, $6.3 \%$ divorced and $1.5 \%$ widowed. $86.8 \%$ reported heterosexuality. For education and income level, $25.2 \%$ reported a highest education level was lower than high school and $16.3 \%$ earned less than an average of 3000 yuan each month.

Six sociodemographic variables (age, gender, education, income, marital status, residence status) were significantly associated with depression. Participants younger than 25 years old were about 4 times $(\mathrm{OR}=4.12,95 \% \mathrm{CI}=2.03-8.37)$ more susceptible to depression than those older than 60 . Women have a higher risk of depression than men $(\mathrm{OR}=1.65,95 \% \mathrm{CI}=1.17-2.34)$. Compared to married group, unmarried status is a risk factor of depression $(\mathrm{OR}=1.87$, $95 \% \mathrm{CI}=1.30-2.68)$. The groups of above 6001 yuan per month was less likely to be depressed compared with the lowest group, and the lower the education level, the greater the likelihood of depression. Non-local people rose the possibility of depression approximately 2 times $(\mathrm{OR}=1.75$, $95 \% \mathrm{CI}=1.23-2.50$ ) higher than local residents.

\section{Mental Health problems and their Correlation with Depression among STIs Patients}

Patients' psychosocial health status is demonstrated in Table 2. Of the 910 participants, $17.9 \%$ reported having had depressive symptoms. Meanwhile, $9.6 \%$ was grouped as low self-esteem and $26.0 \%$ perceived inadequate social support. When using the 75 th percentile of the scale scores as the cut points, $28.9 \%$ felt significantly lonely, $25.9 \%$ and $26.7 \%$ reported entrapment and defeat, respectively, and $25.5 \%$ had dissatisfied interpersonal needs.

Table 3 depicted the correlation between depression and other mental health problems. As seen in the table 3 , all our selected mental health problems showed significant relationships with depression, among which entrapment presented the strongest association with depression $(\mathrm{r}=$ $0.71, \mathrm{P}<0.01)$. Loneliness $(\mathrm{r}=0.48, \mathrm{P}<0.01)$, entrapment, defeat $(\mathrm{r}=0.60, \mathrm{P}<0.01)$ and interpersonal needs $(\mathrm{r}=0.34, \mathrm{P}<0.01)$ were positively correlated with depression, whereas selfesteem $(r=-0.38, P<0.01)$ and social support $(r=-0.16, P<0.01)$ were negatively related with depression.

\section{Mental Health problems associated with depression}

The results of binary regression were shown in Table 4 . After adjusting for age, gender, education, income, marital status, residence status, all psychosocial variables included were

Peerj reviewing PDF | (2021:04:59804:2:0:NEW 17 Jul 2021) 
262

263

264

265

266

267

268

269

270

271

272

273

274

275

276

277

278

279

280

281

282

283

284

285

286

287

288

289

290

291

292

293

294

295

296

297

298

299

300

301

302

303

304

305

statistically significant with depression. The low levels of self-esteem (adjusted odds ratio [AOR]: 6.74, 95\% CI: 4.08-11.14) and social supports (AOR:1.73, 95\% CI: 1.18-2.54), the high levels of loneliness (AOR: 5.75, 95\% CI: 3.96-8.36), entrapment (AOR: 14.80, 95\% CI: 9.6922.59), defeat (AOR: 12.04, 95\% CI: 7.87-18.44), interpersonal needs (AOR: 3.35, 95\% CI: 2.28-4.92) were at elevated risk for depression. However, the multivariate logistic regression only remained four significant variables: self-esteem (Odds ratios obtained from forward stepwise multivariate logistic regression using significant variables of the univariate analysis as input [ORm]: 2.18, 95\% CI: 1.19-4.00), social supports (ORm: 2.18, 95\% CI: 1.37-3.46), entrapment (ORm: 6.31, 95\% CI: 3.75-10.62), defeat (ORm: 2.60, 95\% CI: 1.51-4.48).

\section{Verification of the Syndemic Effect of mental health problems}

The results of the associations between the number of syndemic condition and depression are presented in table 5. It was found that having two or more psychosocial problems at the same time $(37 \%, 337 / 910)$ magnified effect in fusing depression (AOR:11.94, 95\% CI: 7.70-18.53).

The participants were further divided into non-syndemic group, low-level syndemic group (have two to three psychosocial problems) and high-level syndemic group (have four or more psychosocial problems). The low-level group (AOR: 7.52, 95\% CI: 4.60-12.28) and high-level group (AOR: 22.12, 95\% CI: 13.19-37.09) showed a prominent syndemic effect compared with those in the non-syndemic group.

\section{Discussion}

The rate of depression of STIs patients in our study is $17.9 \%$. Compared with the prevalence of depression with $5.1 \%$ and $3.6 \%$ in ordinary women and men, respectively, the depression status of STIs patients deserve more attention (WHO 2017). The concurrence of two or more mental health problems with a high prevalence rate of $37 \%$ demonstrated the syndemic effect to magnify the depressive symptoms of the population. The more psychosocial problems STIs patients have, the more likely they are to have depression. The associations between mental health issues may play important roles in sydemic effect on depression.

We have found 6 possible sociodemographic factors contributing to depression among STIs patients. The depression rate of female patients is much higher than that of male patients $(21.4 \%$ versus $14.1 \%, \mathrm{P}=0.004)$, which is consistent with an American study $(51.9 \%$ versus $31.9 \%, \mathrm{p}=$ 0.023) (Erbelding et al. 2001). Also, women had higher prevalence of STIs and depression than men in Canada (Chen et al. 2008). Unemployment and worsen relationship with sex partner after STIs diagnosis in female patients were risk factors of probable depression (Mo et al. 2015). How age got to become negative factor may be explained by higher sexual impulsivity, less awareness of the disease, lower ability to ask for help and higher vulnerability to events in the younger age group. Emotional and financial support from families and friends may explain married status and local residence as protective factors in depression development. Education degree and its related income levels, affecting medical resource, professional consultant and cost of medication can also be blocks to psychosocial health.

In traditional belief, sexually transmitted infections are considered as social stigma and a shame not only to individual but also to the family (Lichtenstein et al. 2005). The consequent stress as well as physical discomfort may provoke poor mental well-being (Duncan et al. 2001). In our study, all 6 psychosocial variables were related to depression, especially self-esteem,

Peer] reviewing PDF | (2021:04:59804:2:0:NEW 17 Jul 2021) 
306

307

308

309

310

311

312

313

314

315

316

317

318

319

320

321

322

323

324

325

326

327

328

329

330

331

332

333

334

335

336

337

338

339

340

341

342

343

344

345

346

347

348

349

350

351 social support, entrapment and defeat play significant roles in increasing the risk of depression. Self-esteem and social support have been regarded as important resources to fight against stress and to maintain health (Thoits 2010). According to the vulnerability model of depression (Orth \& Robins 2013), lower self-esteem can put individuals at a higher risk of depression when confronting significant stressors . Consistent with the vulnerability model, it has been shown in a meta-analysis of 95 longitudinal studies that low self-esteem carried significantly stronger effect on depression $(\beta=-0.16)$ (Sowislo \& Orth 2013). Studies had also verified the perception of social support (available assistance, receiving actual help and integration in a social work) can prevent the risk of depression(Liu et al. 2016; Maeda et al. 2013; Sonnenberg et al. 2013). Usage of Internet is encouraged by a study on social media who discovered that the more social support people thought they perceived from social media, the less likely they would be trapped in depression (Park et al. 2016). The social support intervention by social media can be carried out in STIs patients to ease the mental health burden.

The results found, in agreement with previous studies, that entrapment and defeat were important predictors of depression, which was also applicable to STIs patients. The concept of entrapment (an inability to escape from adverse situations) and defeat (failed social struggle) derived from the social rank theory of depression (Gilbert \& Allan 1998). Many of evolutionary theories noted that "failed struggle" can be considered as the essence of depression onset. The feelings of entrapment and defeat can emerge from unacceptable or relatively novel issues, social threat, stressors. To our knowledge, these are common problems encountered by STIs patients. The previous studies have shown clear and robust correlations between entrapment, defeat and depression (Gilbert \& Allan 1998; Griffiths et al. 2014; Taylor et al. 2011; Trachsel et al. 2010). Furthermore, a previous systematic review reported that self-perceived defeat and entrapment played key roles in depression, anxiety, suicidal tendency, and post-traumatic stress disorder, and emphasized that entrapment played a decisive role in depression (Siddaway et al. 2015). There are implications for targeting perceptions of entrapment and defeat within psychosocial interventions for STIs patients suffering from depression and screening the population to identify those at risk of developing depression.

The most significant finding of the study is that psychosocial syndemic revealed a magnifying effect in predicting depression status of STIs patients. The interaction between psychosocial variables leads to the coexistence of psychosocial problems. The self-esteem antecedent model argues that self-esteem has an effect on social support. Specifically, higher self-esteem can get individuals to develop positive social support networks because of their belief in their social worth, while those with lower self-esteem have difficulties in establishing positive social support systems because they usually avoid social interaction due to their worry about being rejected by others (Marshall et al. 2014). On the other hand, the self-esteem consequence model points out that positive social support can produce higher self-esteem(Leary 2005). The mediation effect of social support on loneliness and depression can shed light on the concurrence effects of loneliness and social support on depression (Liu et al. 2016). In addition, some researcher conducted factor analysis to verify entrapment and defeat were best defined as on factor, suggesting that the perceptions cooccurred and lead to development of mental disorders (Griffiths et al. 2015; Griffiths et al. 2014). Therefore, we integrated various mental health issues and formed a comprehensive framework for the preliminary evaluation of depression among STIS patients. As a conclusion, a high number of psychosocial problems indicated a greater potentiality of depression.

Peer] reviewing PDF | (2021:04:59804:2:0:NEW 17 Jul 2021) 
352

353

354

355

356

357

358

359

360

361

362

363

364

365

366

367

368

369

370

371

372

373

374

375

376

377

378

379

380

381

382

383

384

385

386

387

388

389

390

391

\section{Limitations}

This survey had several limitations. First, analysis of cross-sectional result alone is hard to figure out the causality thus a prospective study assessing the cause-and-effect relationships between psychosocial variables and depression is needed in the future. Second, the data in this study was only from Shanghai Skin Disease Hospital and obtained by convenient sampling method, so multicenter research nationwide with random sampling method is expected to further generalize the conclusion. Third, despite regular training and active feedback with interviewers, the bias of recall and self-report from interviewees were unavoidable. Fourth, some psychosocial problems related to STIs patients such as stigma and stress were not taken into consideration. Finally, there is insufficient evidence to offer generally recognized cut-offs to divide high-level and low-level groups of psychosocial variables.

\section{Conclusions}

Our study reviewed the contribution of each psychosocial variable to the development of depression in sexually transmitted infections patients and extended the literature into the study. The correlation between psychosocial problems and depression was confirmed, and a syndemic effect of psychosocial problems on increasing odds of depression was identified. We emphasize the importance of early and integrated psychosocial intervention to alleviate their mental burden so that the occurrence and progression of depression can be blocked.

\section{Acknowledgements}

We are grateful to the individuals who volunteered their time to participate the study.

\section{Declaration of Competing Interest}

The authors declare that they have no conflicts of interest to report.

\section{Funding}

This work was supported by the National Key R\&D Program of China (Grant Nos. 2020YFC2006400), Shanghai Three-year Action Plan for Public Health under Grant GWV-10.2XD13, GWV-10.1-XK15, GWV-10.1-XK18, and by Strategic collaborative innovation team (SSMU-ZLCX20180601). The funding body played no role in the design of the study and collection, analysis, and interpretation of data and in writing the manuscript.

\section{References}

Carrera SG, and Wei M, et al. 2017. Thwarted belongingness, perceived burdensomeness, and depression among asian americans: A longitudinal study of interpersonal shame as a mediator and perfectionistic family discrepancy as a moderator. J Couns Psychol 64:280291. 10.1037/cou0000199

Carvalho S, Pinto-Gouveia J, Pimentel P, Maia D, Gilbert P, and Mota-Pereira J, et al. 2013. Entrapment and defeat perceptions in depressive symptomatology: through an evolutionary 
approach. Psychiatry 76:53-67. 10.1521/psyc.2013.76.1.53

393

394

395

396

397

398

399

400

401

402

403

404

405

406

407

408

409

410

411

412

413

414

415

416

417

418

419

420

421

422

423

424

425

426

427

428

Chen X, et al. 2019. Historical perspective on prevention and control of sexually transmitted disease in the past 70 years in China: progress and achievement International Journal of Epidemiology and Infectious Disease 46. 10.3760/cma.j.issn.1673-4149.2019.04.001

Chen Y, Wu J, Yi Q, Huang G, and Wong T, et al. 2008. Depression associated with sexually transmitted infection in Canada. Sex Transm Infect 84:535-540. 10.1136/sti.2007.029306

Cheung PC, and Lau S, et al. 1985. Self-esteem--its relationship to the family and school social environments among Chinese adolescents. Youth \& Society 16:438-456.

Choi Y, Choi SH, Yun JY, Lim JA, Kwon Y, Lee HY, and Jang JH, et al. 2019. The relationship between levels of self-esteem and the development of depression in young adults with mild depressive symptoms. Medicine (Baltimore) 98:e17518. 10.1097/md.0000000000017518

Domènech-Abella J, Mundó J, Haro JM, and Rubio-Valera M, et al. 2019. Anxiety, depression, loneliness and social network in the elderly: Longitudinal associations from The Irish Longitudinal Study on Ageing (TILDA). $J$ Affect Disord 246:82-88. 10.1016/j.jad.2018.12.043

Duncan B, Hart G, Scoular A, and Bigrigg A, et al. 2001. Qualitative analysis of psychosocial impact of diagnosis of Chlamydia trachomatis: implications for screening. Bmj 322:195199. $10.1136 / \mathrm{bmj} .322 .7280 .195$

Erbelding EJ, Hummel B, Hogan T, and Zenilman J, et al. 2001. High rates of depressive symptoms in STD clinic patients. Sex Transm Dis 28:281-284. 10.1097/00007435200105000-00008

Gilbert P, and Allan S, et al. 1998. The role of defeat and entrapment (arrested flight) in depression: an exploration of an evolutionary view. Psychol Med 28:585-598.

Griffiths AW, Wood AM, Maltby J, Taylor PJ, Panagioti M, and Tai S, et al. 2015. The development of the Short Defeat and Entrapment Scale (SDES). Psychol Assess 27:11821194. $10.1037 /$ pas0000110

Griffiths AW, Wood AM, Maltby J, Taylor PJ, and Tai S, et al. 2014. The prospective role of defeat and entrapment in depression and anxiety: a 12-month longitudinal study. Psychiatry Res 216:52-59. 10.1016/j.psychres.2014.01.037

Guadamuz TE, McCarthy K, Wimonsate W, Thienkrua W, Varangrat A, Chaikummao S, Sangiamkittikul A, Stall RD, and van Griensven F, et al. 2014. Psychosocial health conditions and HIV prevalence and incidence in a cohort of men who have sex with men in Bangkok, Thailand: evidence of a syndemic effect. AIDS Behav 18:2089-2096. 10.1007/s10461-014-0826-8

Han JW, and Kim DJ, et al. 2020. Longitudinal Relationship Study of Depression and Self-Esteem in Postnatal Korean Women Using Autoregressive Cross-Lagged Modeling. Int J Environ Res Public Health 17. 10.3390/ijerph17103743 
429 Hays RD, and DiMatteo MR, et al. 1987. A short-form measure of loneliness. J Pers Assess 51:6981. 10.1207/s15327752jpa5101_6

431

432

433

434

435

436

437

438

439

440

441

442

443

444

445

446

447

448

449

450

451

452

453

454

455

456

457

458

459

460

461

462

463

464

465

$\mathrm{Hu}$, and Ai H, et al. 2016. Self-esteem mediates the effect of the parent-adolescent relationship on depression. J Health Psychol 21:897-904. 10.1177/1359105314541315

Hua Tang SW, Ruijie Gong, Zezhou Wang, Yong Cai, et al. 2019. Reliability and validity of defeat scale on anxiety and depression in medical students. Journal of Shanghai Jiaotong University(Medical Science) 39:84-88. (in Chinese)

Huang SY, Hung JH, Hu LY, Huang MW, Lee SC, and Shen CC, et al. 2018. Risk of sexually transmitted infections following depressive disorder: A nationwide population-based cohort study. Medicine (Baltimore) 97:e12539. 10.1097/md.0000000000012539

Hutton HE, Lyketsos CG, Zenilman JM, Thompson RE, and Erbelding EJ, et al. 2004. Depression and HIV risk behaviors among patients in a sexually transmitted disease clinic. Am J Psychiatry 161:912-914. 10.1176/appi.ajp.161.5.912

Kroenke K, Spitzer RL, and Williams JB, et al. 2001. The PHQ-9: validity of a brief depression severity measure. J Gen Intern Med 16:606-613. 10.1046/j.1525-1497.2001.016009606.x

Kroenke K, Spitzer RL, Williams JB, and Löwe B, et al. 2010. The Patient Health Questionnaire Somatic, Anxiety, and Depressive Symptom Scales: a systematic review. Gen Hosp Psychiatry 32:345-359. 10.1016/j.genhosppsych.2010.03.006

Leary MR, et al. 2005. Sociometer theory and the pursuit of relational value: Getting to the root of self-esteem. . European Review of Social Psychology 16:75-111. $10.1080 / 10463280540000007$

Li R, Cai Y, Wang Y, Sun Z, Zhu C, Tian Y, Jiang X, and Gan F, et al. 2016. Psychosocial syndemic associated with increased suicidal ideation among men who have sex with men in Shanghai, China. Health Psychol 35:148-156. 10.1037/hea0000265

Lichtenstein B, Hook EW, 3rd, and Sharma AK, et al. 2005. Public tolerance, private pain: stigma and sexually transmitted infections in the American Deep South. Cult Health Sex 7:43-57. $10.1080 / 13691050412331271416$

Liu L, Gou Z, and Zuo J, et al. 2016. Social support mediates loneliness and depression in elderly people. J Health Psychol 21:750-758. 10.1177/1359105314536941

Maeda U, Shen BJ, Schwarz ER, Farrell KA, and Mallon S, et al. 2013. Self-efficacy mediates the associations of social support and depression with treatment adherence in heart failure patients. Int J Behav Med 20:88-96. 10.1007/s12529-011-9215-0

Manea L, Gilbody S, and McMillan D, et al. 2015. A diagnostic meta-analysis of the Patient Health Questionnaire-9 (PHQ-9) algorithm scoring method as a screen for depression. Gen Hosp Psychiatry 37:67-75. 10.1016/j.genhosppsych.2014.09.009

Marshall SL, Parker PD, Ciarrochi J, and Heaven PCL, et al. 2014. Is self-esteem a cause or consequence of social support? A 4-year longitudinal study. Child Dev 85:1275-1291. 
466

467

468

469

470

471

472

473

474

475

476

477

478

479

480

481

482

483

484

485

486

487

488

489

490

491

492

493

494

495

496

497

498

499

500

501

502

503

10.1111/cdev.12176

Mo PK, Gu J, Lau JT, and You H, et al. 2015. Prevalence and associated factors of mental health problems among monogamous Chinese female patients with sexually transmitted diseases in Hong Kong. AIDS Care 27:481-488. 10.1080/09540121.2014.972321

Orth U, and Robins RW, et al. 2013. Understanding the Link Between Low Self-Esteem and Depression. Current Directions in Psychological Science 22:455-460. $10.1177 / 0963721413492763$

Park J, Lee DS, Shablack H, Verduyn P, Deldin P, Ybarra O, Jonides J, and Kross E, et al. 2016. When perceptions defy reality: The relationships between depression and actual and perceived Facebook social support. J Affect Disord 200:37-44. 10.1016/j.jad.2016.01.048

Polat B, Orhan KS, Kesimli MC, Gorgulu Y, Ulusan M, and Deger K, et al. 2015. The effects of indwelling voice prosthesis on the quality of life, depressive symptoms, and self-esteem in patients with total laryngectomy. Eur Arch Otorhinolaryngol 272:3431-3437. $10.1007 / \mathrm{s} 00405-014-3335-5$

Qi SZ, Wang SM, Shi JF, Wang QQ, Chen XS, Sun LJ, Liu A, Zhang N, Jiang N, Siva P, Xu XL, and Qiao YL, et al. 2014. Human papillomavirus-related psychosocial impact of patients with genital warts in China: a hospital-based cross-sectional study. BMC Public Health 14:739. 10.1186/1471-2458-14-739

Rosenberg M, et al. 1965. Society and the Adolescent Self-Image. Princeton: Princeton University Press.

Rowley J VHS, Korenromp E, Low N, Unemo M, Abu-Raddad LJ, et al. June 2019. Global and Regional Estimates of the Prevalence and Incidence of Four Curable Sexually Transmitted Infections in 2016. WHO Bulletin.

Ruijie Gong JL, Yichen Wang, Yong Cai, Suping Wang, et al. 2019. Validity and reliability of the Chinese vision of the Entrapment Scale in medical students. Chinese Mental Health Journal 33:393-397. (in Chinese)

Shrier LA, Harris SK, and Beardslee WR, et al. 2002. Temporal associations between depressive symptoms and self-reported sexually transmitted disease among adolescents. Arch Pediatr Adolesc Med 156:599-606. 10.1001/archpedi.156.6.599

Siddaway AP, Taylor PJ, Wood AM, and Schulz J, et al. 2015. A meta-analysis of perceptions of defeat and entrapment in depression, anxiety problems, posttraumatic stress disorder, and suicidality. J Affect Disord 184:149-159. 10.1016/j.jad.2015.05.046

Sinclair SJ, Blais MA, Gansler DA, Sandberg E, Bistis K, and LoCicero A, et al. 2010. Psychometric properties of the Rosenberg Self-Esteem Scale: overall and across demographic groups living within the United States. Eval Health Prof 33:56-80. $10.1177 / 0163278709356187$

Sonnenberg CM, Deeg DJ, van Tilburg TG, Vink D, Stek ML, and Beekman AT, et al. 2013. Gender differences in the relation between depression and social support in later life. Int

Peer] reviewing PDF | (2021:04:59804:2:0:NEW 17 Jul 2021) 
504

505

506

507

508

509

510

511

512

513

514

515

516

517

518

519

520

521

522

523

524

525

526

527

528

529

530

531

532

533

534

535

536

537

538

539

Psychogeriatr 25:61-70. 10.1017/s1041610212001202

Sowislo JF, and Orth U, et al. 2013. Does low self-esteem predict depression and anxiety? A metaanalysis of longitudinal studies. Psychol Bull 139:213-240. 10.1037/a0028931

Spitzer RL, Kroenke K, and Williams JB, et al. 1999. Validation and utility of a self-report version of PRIME-MD: the PHQ primary care study. Primary Care Evaluation of Mental Disorders. Patient Health Questionnaire. Jama 282:1737-1744. 10.1001/jama.282.18.1737

Taylor PJ, Gooding P, Wood AM, and Tarrier N, et al. 2011. The role of defeat and entrapment in depression, anxiety, and suicide. Psychol Bull 137:391-420. 10.1037/a0022935

Thoits PA, et al.. 2010. Stress and health: major findings and policy implications. J Health Soc Behav 51 Suppl:S41-53. 10.1177/0022146510383499

Trachsel M, Krieger T, Gilbert P, and Grosse Holtforth M, et al. 2010. Testing a german adaption of the entrapment scale and assessing the relation to depression. Depress Res Treat 2010:501782. 10.1155/2010/501782

Tsai AC, et al. 2018. Syndemics: A theory in search of data or data in search of a theory? Soc Sci Med 206:117-122. 10.1016/j.socscimed.2018.03.040

Van Orden KA, Witte TK, Gordon KH, Bender TW, and Joiner TE, Jr, et al. 2008. Suicidal desire and the capability for suicide: tests of the interpersonal-psychological theory of suicidal behavior among adults. J Consult Clin Psychol 76:72-83. 10.1037/0022-006x.76.1.72

Wang S, Ni Y, Gong R, Shi Y, Cai Y, and Ma J, et al. 2020. Psychosocial Syndemic of suicidal ideation: a cross-sectional study among sexually transmitted infection patients in Shanghai, China. BMC Public Health 20:1314. 10.1186/s12889-020-09404-y

Wang W, Xiao C, Yao X, Yang Y, Yan H, and Li S, et al. 2018. Psychosocial health and suicidal ideation among people living with HIV/AIDS: A cross-sectional study in Nanjing, China. PLoS One 13:e0192940. 10.1371/journal.pone.0192940

WHO. 2017. Depression and Other Common Mental Disorders, Global Health Estimates.

WHO. 2020. Home/Newsroom/Fact sheets/Detail/Depression. Available at https://www.who.int/news-room/fact-sheets/detail/depression.

Xiaomin Li TX, Jing Yuan, Lixia Lv, Jiayu Tao, Yong Liu, et al. 2015. Validity and Reliability of the Interpersonal Needs Questionnaire in Chinese College Students. Chinese Journal of Clinical Psychology 23:590-593. (in Chinese)

Xing-Chen HU, Ying-Li Z, Wei L, Hong-Mei Z, and Shi-Chang Y, et al. 2014. Reliability and validity of the patient health questionnaire-9 in Chinese adolescents. Sichuan Mental Health. (in Chinese)

Ye X, Liu J, and Yi Z, et al. 2019. Trends in the Epidemiology of Sexually Transmitted Disease, Acquired Immune Deficiency Syndrome (AIDS), Gonorrhea, and Syphilis, in the 31 Provinces of Mainland China. Med Sci Monit 25:5657-5665. 10.12659/msm.915732

Peer] reviewing PDF | (2021:04:59804:2:0:NEW 17 Jul 2021) 
540 Yuan Chen HM, Zi Chen, Yuling Jia, Xiao Wang, Jiaojiao Chen, et al. 2018. Reliability and

541

542

543

544

545

546

547
Validity of Chinese Version of Multidimensional Scale of Perceived Social Support in Elderly People with Chronic Diseases. Journal of Nursing 25:5-8. (in Chinese)

Zhou L, Li Z, Hu M, and Xiao S, et al. 2012. Reliability and validity of ULS-8 loneliness scale in elderly samples in a rural community. Zhong Nan Da Хие Хие Вао Yі Хие Ban 37:11241128. 10.3969/j.issn.1672-7347.2012.11.008 (in Chinese)

Zimet GD, Dahlem, N. W., Zimet, S. G., \& Farley, G. K. , et al. 1988. The Multidimensional Scale of Perceived Social Support. Journal of Personality Assessment 52:30-41.

548 


\section{Table 1 (on next page)}

Sociodemographic characteristics and their associations with depression among STIS patients in Shanghai, China 
1 Table 1 Sociodemographic characteristics and their associations with depression among STIs

2 patients in Shanghai, China

3

\begin{tabular}{|c|c|c|c|}
\hline \multirow{2}{*}{$\begin{array}{l}\text { Sociodemographic } \\
\text { Characteristics }\end{array}$} & \multirow{2}{*}{ Number (\%) } & \multicolumn{2}{|c|}{ Had depression } \\
\hline & & Number (\%) & ORu $(95 \%$ CI $)$ \\
\hline \multicolumn{4}{|l|}{ Case } \\
\hline Inpatient & $296(32.5)$ & $60(36.8)$ & $1.26(0.89-1.80)$ \\
\hline Outpatient & $614(67.5)$ & $103(63.2)$ & 1 \\
\hline \multicolumn{4}{|l|}{ Age group (years) } \\
\hline$<25$ & $89(9.8)$ & $39(23.9)$ & $4.12(2.03-8.37) *$ \\
\hline $25-40$ & $505(55.5)$ & $70(42.9)$ & $0.85(0.46-1.59)$ \\
\hline $41-59$ & $228(25.1)$ & $40(24.5)$ & $1.13(0.58-2.19)$ \\
\hline$\geq 60$ & $88(9.7)$ & $14(8.6)$ & 1 \\
\hline \multicolumn{4}{|l|}{ Gender } \\
\hline Female & $472(51.9)$ & $101(62.0)$ & $1.65(1.17-2.34) *$ \\
\hline Male & $438(48.1)$ & $62(38.0)$ & 1 \\
\hline \multicolumn{4}{|l|}{ Education } \\
\hline Less than high school & $229(25.2)$ & $52(31.9)$ & $1.78(1.19-2.65) *$ \\
\hline High school & $202(22.2)$ & $43(26.4)$ & $1.64(1.07-2.50) *$ \\
\hline College degree or above & $479(52.6)$ & $68(41.7)$ & 1 \\
\hline \multicolumn{4}{|l|}{ Income (RMB) } \\
\hline$\geq 120001$ & $201(22.1)$ & $16(9.8)$ & $0.23(0.12-0.42) *$ \\
\hline $6001-12000$ & $259(28.5)$ & $41(25.2)$ & $0.49(0.30-0.80) *$ \\
\hline
\end{tabular}




$\begin{array}{llll}\text { Syphilis } & 365(40.1) & 68(41.7) & 1.15(0.25-5.35) \\ \text { Genital warts } & 361(39.7) & 62(38.0) & 1.04(0.22-4.85) \\ \text { Genital herpes } & 28(3.1) & 7(4.3) & 1.67(0.29-9.52) \\ \text { Others } & 114(12.5) & 21(12.9) & 1.13(0.23-5.54) \\ \text { HIV } & 12(1.3) & 2(1.2) & 1\end{array}$

4 ORu: Univariate odds ratios

$5 * \mathrm{P}<0.05$

6

7 
Table 2 (on next page)

Frequency Distributions of Psychosocial Variables among STIs Patients in Shanghai, China 
1 Table 2 Frequency Distributions of Psychosocial Variables among STIs Patients in Shanghai,

2 China

3

4

5

6

7

8

9

Psychosocial Variables

Number (\%)

Had depression

Number (\%)

Depression

High level (score $\geq 10$ )

$163(17.9)$

Low level $($ score $<10)$

$747(82.1)$

Self-Esteem

Low level (score $<15)$

$87(9.6)$

$50(30.7)$

High level (score $\geq 15$ )

$823(90.4)$

$113(69.3)$

\section{Social Support}

Low level $($ score $<59)$

$237(26.0)$

$62(38.0)$

High level (score $\geq 59$ )

$673(74.0)$

$101(62.0)$

\section{Loneliness}

High level (score $\geq 18$ )

$263(28.9)$

$100(61.3)$

Low level $($ score $<18)$

647 (71.1)

63 (38.7)

\section{Entrapment}

High level (score $\geq 20$ )

$236(25.9)$

$119(73.0)$

Low level $($ score $<20)$

$674(74.1)$

$44(27.0)$

Defeat

High level (score $\geq 23$ )

$243(26.7)$

$113(69.3)$

Low level $($ score $<23$ )

667 (73.3)

$50(30.7)$

Interpersonal Needs

High level (score $\geq 49$ )

$232(25.5)$

78 (47.9)

Low level (score $<49$ )

$678(74.5)$

$85(52.1)$ 


\section{Table 3 (on next page)}

Descriptive Analysis of Psychosocial Variables and Their Correlations with Depression among STIs Patients in Shanghai, China 
1 Table 3 Descriptive Analysis of Psychosocial Variables and Their Correlations with 2 Depression among STIs Patients in Shanghai, China

3

\begin{tabular}{|c|c|c|c|c|c|c|c|c|c|}
\hline Variables & Mean (SD) & $\begin{array}{l}\text { Median } \\
\text { (IQR) }\end{array}$ & 1 & 2 & 3 & 4 & 5 & 6 & 7 \\
\hline 1.Depression & $6.19(5.39)$ & $5(7)$ & 1 & & & & & & \\
\hline 2. Self-esteem & $19.8(4.64)$ & $19(6)$ & $-0.38 *$ & 1 & & & & & \\
\hline 3. Loneliness & $14.72(4.88)$ & $14(8)$ & $0.48 *$ & $-0.50 *$ & 1 & & & & \\
\hline 4. Social Support & $65.48(14.67)$ & $69(17)$ & $-0.16^{*}$ & $0.33^{*}$ & $-0.41 *$ & 1 & & & \\
\hline 5. Entrapment & $12.12(13.30)$ & $7.5(20)$ & $0.71 *$ & $-0.38^{*}$ & $0.53 *$ & $-0.23 *$ & 1 & & \\
\hline 6. Defeat & $16.50(11.15)$ & $14(15)$ & $0.60 *$ & $-0.61^{*}$ & $0.60 *$ & $-0.30 *$ & $0.38 *$ & 1 & \\
\hline 7. Interpersonal needs & $36.33(14.63)$ & $35(25)$ & $0.34^{*}$ & $-0.54^{*}$ & $0.57 *$ & $-0.42 *$ & $0.42 *$ & $0.58 *$ & 1 \\
\hline
\end{tabular}

$4 * \mathrm{p}<0.01$

5

6 


\section{Table 4 (on next page)}

Mental Health problems Associated with Depression among STIs Patients in Shanghai, China 
1 Table 4 Mental Health problems Associated with Depression among STIs Patients in 2 Shanghai, China

3

\begin{tabular}{llll}
\hline Mental Health problems & ORu (95\%CI) & AOR (95\%CI) & ORm (95\%CI) \\
\hline Self-Esteem & & & \\
High level & 1 & 1 & 1 \\
Low level & $8.42(5.31-13.57) * *$ & $6.74(4.08-11.14) * *$ & $2.18(1.19-4.00) *$
\end{tabular}

Social Support

High level

Low level

\section{Loneliness}

Low level

High level

Entrapment

Low level

High level

\section{Defeat}

Low level

High level

\section{Interpersonal Needs}

Low level

High level
1

$2.01(1.40-2.87) * *$

1

$1.73(1.18-2.54) * *$

$2.18(1.37-3.46) * *$

1
1

$5.75(3.96-8.36) * *$
$5.69(3.97-8.15) * *$

5.75 (3.96-8.36) 
5 AOR: Odds ratios adjusted for age, gender, education, income, marital status, residence status.

6 ORm: Odds ratios obtained from forward stepwise multivariate logistic regression using 7 significant variables of the univariate analysis as input.

$8 \quad * \mathrm{p}<0.05 ; * * \mathrm{p}<0.01$

9

10

11

12

13

14 


\section{Table 5 (on next page)}

Associations Between the Number of Syndemic Condition and Depression among STIs Patients in Shanghai, China 
1 Table 5 Associations Between the Number of Syndemic Condition and Depression among 2 STIs Patients in Shanghai, China

3

\begin{tabular}{llll}
\hline Number & \multicolumn{2}{l}{ Had depression } \\
\cline { 3 - 3 }$(\%)$ & Number & AOR (95\%CI) \\
& $(\%)$ & \\
& & & \\
\hline
\end{tabular}

Have a syndemic

No (have no more than one $573(63.0) \quad 31(19.0) \quad 1$

psychosocial problem)

Yes (have two or more $337(37.0) \quad 132(81.0) \quad 11.94(7.70-18.53) * *$

psychosocial problems)

Number of syndemic conditions

No (have no more than one $573(63.0) \quad 31(19.0) \quad 1$

psychosocial problem)

Low level (have two to three $201(22.1) \quad 59(36.2) \quad 7.52(4.60-12.28) * *$

psychosocial problems)

High level (have four or more 136(14.9) $73(44.8) \quad 22.12(13.19-37.09) * *$ psychosocial problems)

4 AOR: Odds ratios adjusted for age, gender, education, income, marital status, residence status.

$5 * * \mathrm{p}<0.01$

6 